\title{
Spatiotemporal nature of Fusarium graminearum-wheat coleoptile interactions
}

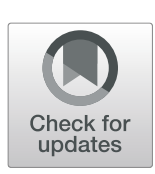

\author{
Han Qiu ${ }^{1 \dagger}$, Xu Zhao ${ }^{1 \dagger}$, Wenqin Fang ${ }^{1}$, Huiming Wu², Yakubu Saddeeq Abubakar ${ }^{3}$, Guo-dong Lu ${ }^{1,2}$, \\ Zonghua Wang ${ }^{1,2,4}$ and Wenhui Zheng ${ }^{1,2^{*}}$ (i)
}

\begin{abstract}
Fusarium head blight (FHB) caused by the ascomycete fungus Fusarium graminearum can result in significant crop losses and render the crops harmful to human health due to contamination with mycotoxin. Although the pathogenesis of $F$. graminearum is widely investigated by molecular genetics approaches, detailed studies about its cellular and developmental processes at the initial stages of infection are very limited. We applied live-cell imaging approach to characterize the spatial and temporal development of growing hyphae and plant responses during $F$. graminearum and wheat coleoptile interactions. The present investigation demonstrates that $F$. graminearum uses two strategies to penetrate the host epidermal cells. The pathogen breaks through the host cell wall with appressoria-like structures derived from surficial hyphae, and also with the narrow pegs from thick and bulbous intracellular hyphae. Live cell imaging in the presence of the endocytic tracker FM4-64 showed that the plasma and intermembranes of the invaded wheat coleoptile cells were intact. Invasive hyphae exhibit branching, budding, pseudohyphae-like growth, cell-to-cell spreading ability, and were sealed within a plant membrane, indicating a biotrophic lifestyle of this fungus inside the invaded cells. Time-lapse imaging suggested that there were callose depositions at the plant cell walls in the form of continuous lines and also on the outer linings of the fungal invasive hyphae at colonization stage. In addition, our studies demonstrate that the activation of the toxisomerelated gene (TR/4) requires external stimuli and is spatio-temporally modulated. Generally, this study provides new insights into the colonization strategies and host response features during F. graminearum-plant interactions. Further tracing of cellular details will significantly contribute to our understanding of molecular mechanisms of $F$. graminearum-host interactions.
\end{abstract}

Keywords: Plant-microbe interaction, Fusarium graminearum, Wheat coleoptiles, Host penetration, Biotrophic, Callose

\section{Background}

Fusarium graminearum, a filamentous ascomycete, is a fungal pathogen that infects a wide range of cereal crops including wheat and barley, causing Fusarium head blight (FHB) (Bai and Shaner 2004; Goswami and Kistler 2004). Being one of the most destructive diseases of wheat, FHB attracts the attention of plant pathologists worldwide

\footnotetext{
* Correspondence: wenhuiz@fafu.edu.cn

+Han Qiu and Xu Zhao contributed equally to this work.

'State Key Laboratory of Ecological Pest Control for Fujian and Taiwan Crops, College of Plant Protection, Fujian Agriculture and Forestry University, Fuzhou, China

${ }^{2}$ Fujian Province Key Laboratory of Pathogenic Fungi and Mycotoxins, College of Life Sciences, Fujian Agriculture and Forestry University, Fuzhou, China

Full list of author information is available at the end of the article
}

(Dean et al. 2012; Figueroa et al. 2018). FHB epidemics are recorded frequently in the United States, United Kingdom and China, the management of which requires billions of dollars (McMullen et al. 1997; Dean et al. 2012). The disease results in yield and quality reductions as well as the contamination of the grains with mycotoxins, particularly trichothecenes, making the crops unsafe for consumption (Desjardins and Proctor 2007; Chen et al. 2019). Sources for Fusarium head blight resistance are limited, and fully resistant cultivars to FHB are not yet available and only one resistance locus Fhb1 (containing a debatable resistance gene PFT and a causative gene His/TaHRC) has thus far been cloned in wheat (Rawat et al. 2016; Li et al. 2019; $\mathrm{Su}$ et al. 2019). Therefore, a thorough investigation of the mechanisms involved in F. graminearum proliferation and

(c) The Author(s). 2019 Open Access This article is distributed under the terms of the Creative Commons Attribution 4.0 International License (http://creativecommons.org/licenses/by/4.0/), which permits unrestricted use, distribution, and reproduction in any medium, provided you give appropriate credit to the original author(s) and the source, provide a link to the Creative Commons license, and indicate if changes were made. The Creative Commons Public Domain Dedication waiver (http://creativecommons.org/publicdomain/zero/1.0/) applies to the data made available in this article, unless otherwise stated. 
subsequent colonization of its host cells will help in devising effective means of controlling FHB.

Extensive cellular and ultrastructural analyses have been performed on wheat florets injected with spore suspensions of $F$. graminearum, considering the facts that FHB disease is airborne and it initiates when spores from the pathogen land on flowering spikelets of the host (Parry et al. 1995; Kang and Buchenauer 2000a; Pritsch et al. 2000; Trail 2009). Studies found that $F$. graminearum initially colonises the surface of the wheat florets usually without immediate penetration (Kang and Buchenauer 2000a; Brown et al. 2010). After conidial germination, infectious hyphae become adapted to the intercellular space of the host cells (Kang et al. 2005; Brown et al. 2010). The pathogen also gets into the host plant either through natural openings, like the stomata (Kang and Buchenauer 2000a; Pritsch et al. 2000; Trail 2009), or by penetrating the cell walls by means of infection pegs (Kang and Buchenauer 2000a, b; Kang et al. 2005). F. graminearum, unlike some phytopathogenic fungi (such as Magnaporthe oryzae and Colletotrichum orbiculare), does not require appressoria to penetrate its host cells (Ryder and Talbot 2015; Talbot 2019). However, it produces two specialised infection structures namely foot structures and compound appressoria (including lobate appressoria and infection cushions), on inoculated caryopses, paleas, lemmas and glumes of susceptible and resistant wheat cultivars (Boenisch and Schäfer 2011). After penetration, two kinds of specific invasive hyphal structures (subcuticular hyphae and bulbous infection hyphae) are differentiated during invasion in detached wheat glumes, and they appear to be morphologically distinct from surface hyphae (Rittenour and Harris 2010). At later stages of infection, the pathogen spreads to other florets where it predominately switches from vertical to lateral growths and accumulates below the surface of the rachis, a typical symptom of the head blight of wheat which manifests itself in the form of necrotic lesion (Brown et al. 2010). Despite the delicate microscopic studies that have been conducted, the cytological details of $F$. graminearum-wheat pathosystem are not completely understood.

To study the colonization processes of $F$. graminearum in its hosts, several F. graminearum-host pathosystems have been investigated, using different cytological methods, which include $F$. graminearum-wheat spikes (Kang and Buchenauer 2000a; Pritsch et al. 2000), F. graminearum-barley spikes (Skadsen and Hohn 2004; Jansen et al. 2005), F. graminearum-Arabidopsis thaliana (Skadsen and Hohn 2004), F. graminearum-wheat glumes (Rittenour and Harris 2010), F. graminearumBrachypodium distachyon (Peraldi et al. 2011), F. graminearum-maize (Zhang et al. 2016) and F. graminearum-wheat coleoptile (Wu et al. 2005; Zhang et al. 2012; Jia et al. 2017). These pathosystems, and their respective cytological methods, vastly promoted the understanding of the interactions between $F$. graminearum and its host plants, most notably that of F. graminearum-wheat coleoptile pathosystem. Since the structure of wheat coleoptile is simple and its infection assay could be performed in a growth chamber and its growth is not limited by seasonal, geographical and environmental factors, wheat coleoptiles are most feasible for cytological tracking and time-course transcriptomic analyses of $F$. graminearum invasion processes (Zhang et al. 2012; Jia et al. 2017). Previously, laser capture microdissection and microarray technology have been employed to characterize stage-specific gene expression patterns in wheat coleoptiles during the invasion of $F$. graminearum, and several important virulence genes have been identified (Zhang et al. 2012). In addition, a recently published study had identified a new metabolite fusaoctaxin A as a virulence factor that facilitates $F$. graminearum cell-tocell hyphal progression in wheat coleoptile and is associated with suppression of host cell defense responses (Jia et al. 2019). Despite these achievements, cytological details about $F$. graminearum development on host surfaces and penetration strategies at the initial stages of infection still await future investigations. Furthermore, there are still some unanswered questions regarding inter- and intra-cellular host invasion strategies by $F$. graminearum. Again, it is still unresolved whether $F$. graminearum exhibits biotrophic lifestyle during the initial stages of infection, and also, the spatial and temporal responses of wheat cells after challenge with $F$. graminearum are largely unknown.

In this study, we describe a detailed microscopic investigation of the entire colonisation processes of wheat coleoptile by fluorescently-tagged $F$. graminearum $\mathrm{PH}-1$ strain. In addition, the spatial and temporal patterns of callose deposition by the invaded wheat coleoptile cell walls and toxisome formation during the fungal infection have been documented. Collectively, this study unveils detailed cellular mechanisms and critical strategies involved in successful colonization during $F$. graminearum-plant interaction.

\section{Results}

Early colonisation and development of appressoria-like structures in $F$. graminearum

To study the cytological events in living tissues of the host during F. graminearum infection, we used a live-cell confocal microscopy to observe wheat coleoptile cells invaded by GFP-tagged F. graminearum (Fig. 1). A red fluorescent dye, propidium iodide (PI), was used to visualize the cell wall of the wheat coleoptile. The PI dye 


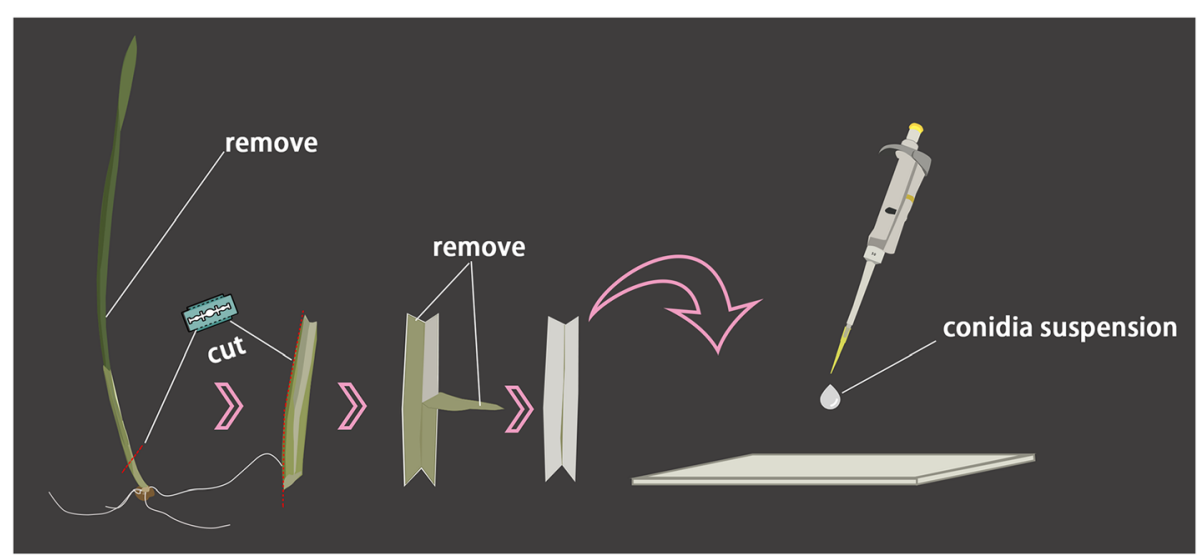

Fig. 1 The processes for wheat coleoptiles inoculation. In short, the bottom of the coleoptile of a 4-day-old wheat seedling was cut off. Next, the epidermis was removed using a sterile surgical blade and mounted on a glass slide. A drop of conidia suspension of F. graminearum was placed on the mounted coleoptile using a micropipette $(0.5-10 \mu \mathrm{L})$, and incubated at $25^{\circ} \mathrm{C}$ and $90 \%$ relative humidity for $1-2$ days

is a small fluorescent molecule that exhibits bright red fluorescence upon binding to DNA. In addition, it has also been used to visualize the outlines (cell walls) of the cells since it does not passively traverse into cells that possess intact plasma membranes (PM) (Crowley et al. 2016; Jones et al. 2016). We therefore reason that simultaneous imaging of the fluorescently-tagged F. graminearum (GFP) inside the PI-stained wheat coleoptile cells could optimize the characterization of the interactions between $F$. graminearum and the wheat coleoptile.

At early stages of the infection (5-8 h post inoculation, hpi), conidia of $F$. graminearum germinated into epiphytic runner hyphae on the extracellular surface or intercellular space of the wheat coleoptiles (Fig. 2). Usually, the runner hyphae do not infect host tissues immediately, but give rise to hyphae that grow and branch on host surfaces. Interestingly, after hyphal branching, we observed that multiple appressoria-like structures of the fungus are formed at the hyphal tips (Fig. 2a, b), consistent with previous observations that appressoria-like structures are developed when intracellular hyphae attack the epicarp cells of wheat and barley (Jansen et al. 2005). In addition, some infectious hyphae could be observed in the intercellular space between the wheat coleoptile cells (Fig. 2c). Upon staining with PI, the clear and intact cellular outlines of the wheat coleoptile cells were detected (Fig. 2c and Additional file 1: Video S1), confirming that the host cells were viable at this stage, and that the hyphae adopt biotrophic lifestyle to invade the host cells.

\section{F. graminearum penetrates the host cell walls with the highly plastic invasion peg}

By $16 \mathrm{hpi}$, we observed that infectious hyphae arising from the surface hyphae began to penetrate the host epidermal cell walls (Fig. 3a, b and Additional file 2:
Video S2). Interestingly, at the penetration site, the penetration peg was markedly constricted (about $1 \mu \mathrm{m})$ as it pierces through the wheat coleoptile cell walls. Upon reaching the epidermal cell lumen, the penetration peg expands to form a normal-sized filamentous hypha, having hyphal widths of about $3 \mu \mathrm{m}$ (Fig. 3a, b and Additional file 2: Video S2). Remarkably, we noticed that the infectious hyphae in the intercellular space of the wheat coleoptiles display significant morphological changes when compared with those arising from the epiphytic surface (Fig. 3c, d). They were thicker and more bulbous; and filled up the intercellular space of the wheat coleoptile cells and subsequently break the cell walls of the wheat coleoptiles to get access into the cells (Fig. 3c, d). We also observed that at certain potential and existing penetration sites, infectious hyphae become slightly swollen prior to sending the highly constricted peg across the cell wall (Fig. 3c), indicating that $F$. graminearum penetrates the wheat cuticles and epidermal walls using highly plastic invasion peg.

\section{Proliferation and cell-to-cell invasion of $F$. graminearum}

After penetrating the wheat coleoptile cells at $16 \mathrm{hpi}$, invasive hyphae were observed to grow in the first invaded cell and often fill it up (Fig. 4). Laser confocal imaging showed that the invasive hyphae exhibit branching, budding and pseudohyphae-like growth instead of the typical filamentous tip growth on media (Fig. 4). These invasive hyphae also display bulbous shape with circular vacuoles, although they appear less bulbous than the runner hyphae in the intercellular space between wheat coleoptile cells (Fig. 4). Similar to the initial penetration, the invasive hyphae in the first-invaded cell continue to spread to the next adjacent cells by means of highly constricted invasive 

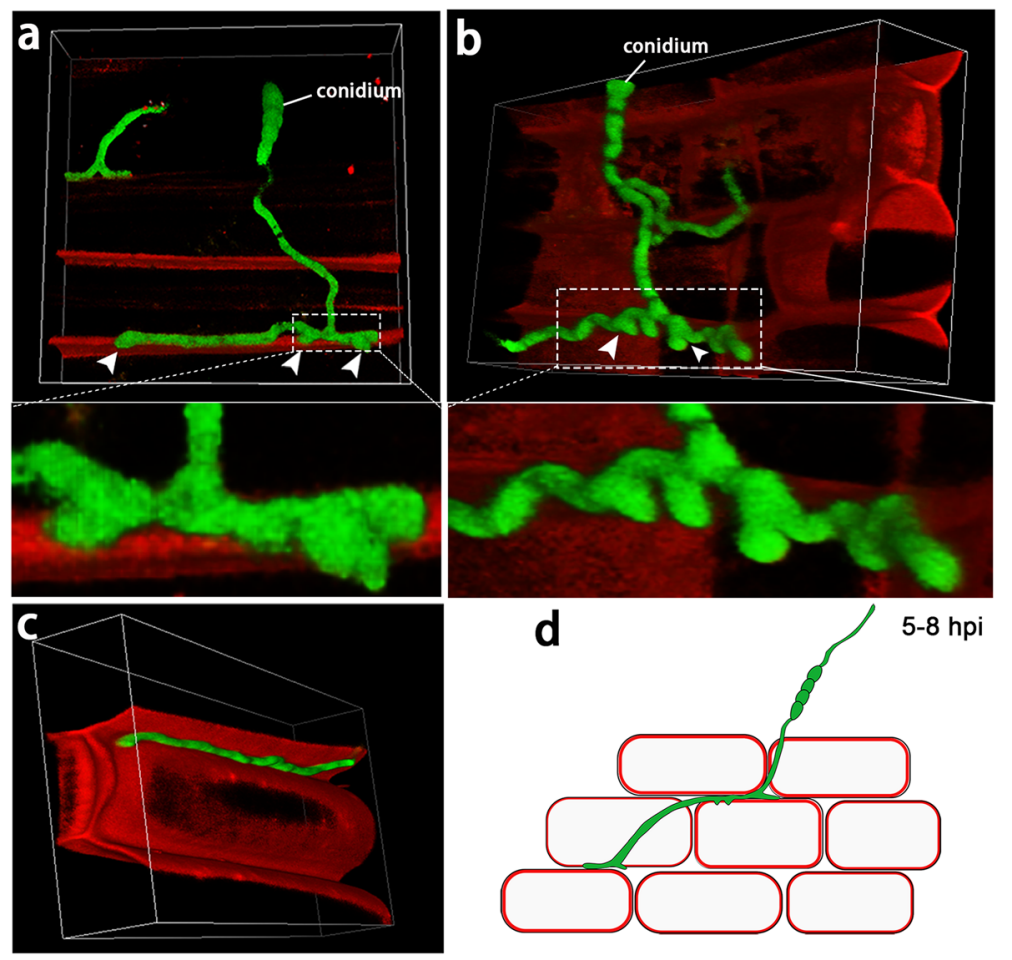

Fig. 2 Laser confocal microscopic images of F. graminearum infection of wheat coleoptiles at 8 hpi. Tissue cells of wheat coleoptiles were stained with PI (Propidium iodide) (red). a, b A conidium of F. graminearum germinated into epiphytic runner hyphae on the extracellular surface of wheat coleoptiles. After hyphal branching, multiple appressoria-like structures are formed at the hyphal tip. Arrowheads indicate appressoria-like structures. Detailed views of each boxed region are shown at the bottom of the respective images. These images are 3D (three-dimensional) reconstructions of 65 optical sections acquired with a z-interval of $0.4 \mu \mathrm{m}$. c A 3D image showing a hypha growing in an intercellular space of a wheat coleoptile cell. Clear and intact cellular outlines were stained by PI, indicating that wheat coleoptile cells were alive at this stage. This image is a 3D reconstruction of 201 optical sections acquired with a z-interval of $0.15 \mu \mathrm{m}$. $\mathbf{d}$ A proposed model depicting the early stages (5-8 hpi) of F. graminearum proliferation inside wheat coleoptiles

hyphal pegs (about $1 \mu \mathrm{m}$ ), and the pegs expand (about $3.65 \mu \mathrm{m})$ and grow as bulbous invasive hyphae in the newly invaded cells (Fig. 4a). Interestingly, most of the second- and later-invaded wheat coleoptile cells did not appear dead at $36 \mathrm{hpi}$ as evidenced by the presence of intact and clearly PI-stained cells. Occasionally, PI staining identifies a few dead invasive hyphae in the infected wheat coleoptile cells (Fig. 4b, d, as indicated by the arrows), suggesting that the plant defense is largely activated at this stage.

\section{F. graminearum uses biotrophic invasion strategy to colonize wheat coleoptile cells}

Since the result of PI staining indicated that the firstand later-invaded wheat coleoptile cells were physically intact after $F$. graminearum infection, we hypothesize that the invasion of the first cell and subsequent invasions of the neighboring cells are biotrophic, an assertion that remains unproven to date. To test this hypothesis, we labeled the plasma membranes of the infected wheat coleoptile cells with the membrane-selective dye FM4-64 (an endocytosis marker which stains plasma membranes, laterally transports into the cells and temporally stains the vacuolar membranes) (Bolte et al. 2004; Kankanala et al. 2007). We observed that the invasive hyphae of $F$. graminearum were clearly outlined by a thin layer of FM4-64 fluorescence (Fig. 5a), suggesting that the plasma membranes of the invaded wheat coleoptile cells were intact and functional at this time. This result is consistent with a previous study in $M$. oryzae-rice pathosystem (Kankanala et al. 2007). In addition, we noticed that some vacuolar membranes of the wheat coleoptile cells have also been labeled by the FM4-64 (Fig. 5, indicated by the arrowheads), suggesting that the invaded wheat coleoptile cells not only have intact internal membrane systems, but also undergo endocytosis. Therefore, these results confirm that the first $F$. graminearum-invaded cell was alive. Furthermore, the results of FM4-64 uptake demonstrate that the invasive hyphae transiting into the neighboring cells were still sheathed within the host plasma membrane (Fig. 5b), indicating that F. graminearum employs biotrophic strategy for its cell-to-cell invasion. 

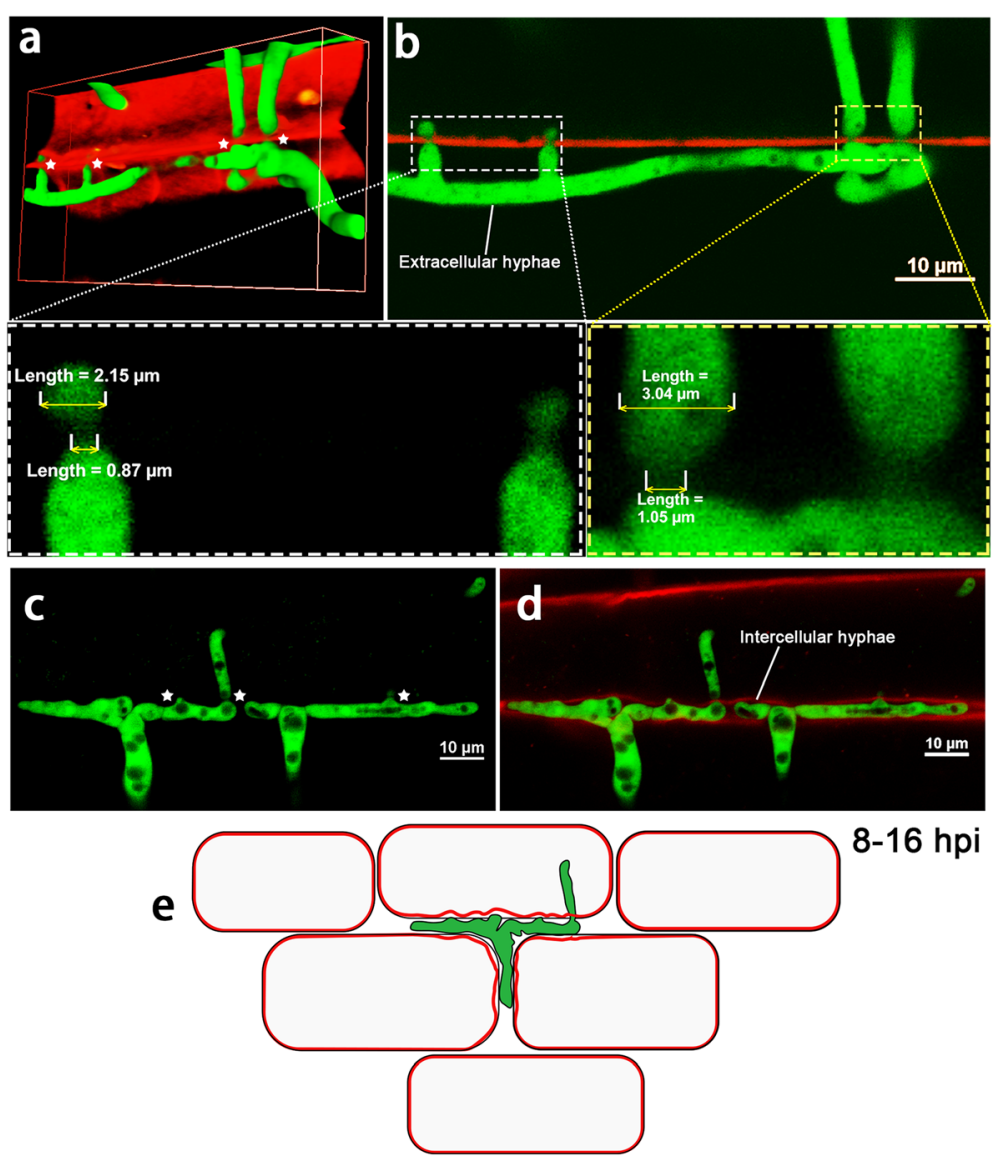

Fig. 3 Laser confocal microscopic images of F. graminearum infection of wheat coleoptiles at 16 hpi. Tissue cells of wheat coleoptiles were stained with PI (Propidium iodide) (red). a A 3D image showing extracellular hyphae traversing the cell wall of wheat coleoptiles through pits. Asterisks indicate penetration sites. This image is a $3 \mathrm{D}$ reconstruction of 31 optical sections acquired with a z-interval of $0.35 \mu \mathrm{m}$. $\mathbf{b}$ Extracellular hyphae began to penetrate the cell wall of wheat coleoptiles at 16 hpi. Detailed views of each boxed region are shown. It could be seen that penetration pegs experience extreme constriction (about $1 \mu \mathrm{m}$ ) as they penetrate the wheat coleoptiles cell walls. $\mathbf{c}$, $\mathbf{d}$ Bulbous and vacuolar hyphae are formed in the intercellular space of wheat coleoptiles at 16 hpi. Potential and existing penetration sites are indicated with asterisks. $\mathbf{e}$ A proposed model depicting the early stages (8-16 hpi) of F. graminearum proliferation inside wheat coleoptiles

\section{F. graminearum colonization leads to callose deposition on the plant cell wall}

In plants, cell walls are one of the first lines of defense that protect the cells from pathogens and are a major factor in basal host resistance (Chowdhury et al. 2014; Malinovsky et al. 2014). Callose are (1,3)-beta-glucans rapidly deposited on plant cell walls when the plants are under stress, such as being invaded by pathogens (Stone et al. 1984; Crowley et al. 2016; Jia et al. 2019). To determine the dynamics of the host defense during F. graminearum invasion, we performed time-course confocal microscopy on wheat coleoptiles infected with GFPtagged PH-1 strain. We stained both the mock and infected tissues with aniline blue, a dye that specifically stains callose and emits visible fluorescence under a fluorescence microscope (Stone et al. 1984; Jia et al. 2019). We observed that the mock-inoculated coleoptile cells were poorly labeled with aniline blue and only showed weak and limited discrete foci at the cell walls (Fig. 6a). However, the treatment group emits the aniline blue signal which accumulates into continuous lines after the F. graminearum penetration (8 hpi) (Fig. 6b), signifying callose deposition. By $16 \mathrm{hpi}$, the callose accumulated dramatically when a hypha begins to traverse the host cell wall (Fig. 6c). Interestingly, with the help of a three-dimensional (3D) reconstruction technique, we noticed the presence of two pores formed on the wheat coleoptile cell wall due to $F$. graminearum peg penetration (Fig. 6c and Additional file 3: Video S3). At 24 hpi, callose was not only deposited between the neighboring cell walls but also aligned on the outer surfaces of the invasive hyphae, due to extensive colonization by $\mathrm{F}$. graminearum (Fig. 6d). Taken together, our results show a spatial and temporal pattern of plant responses via callose deposition on the inner lining of the invaded wheat coleoptile cell walls. 

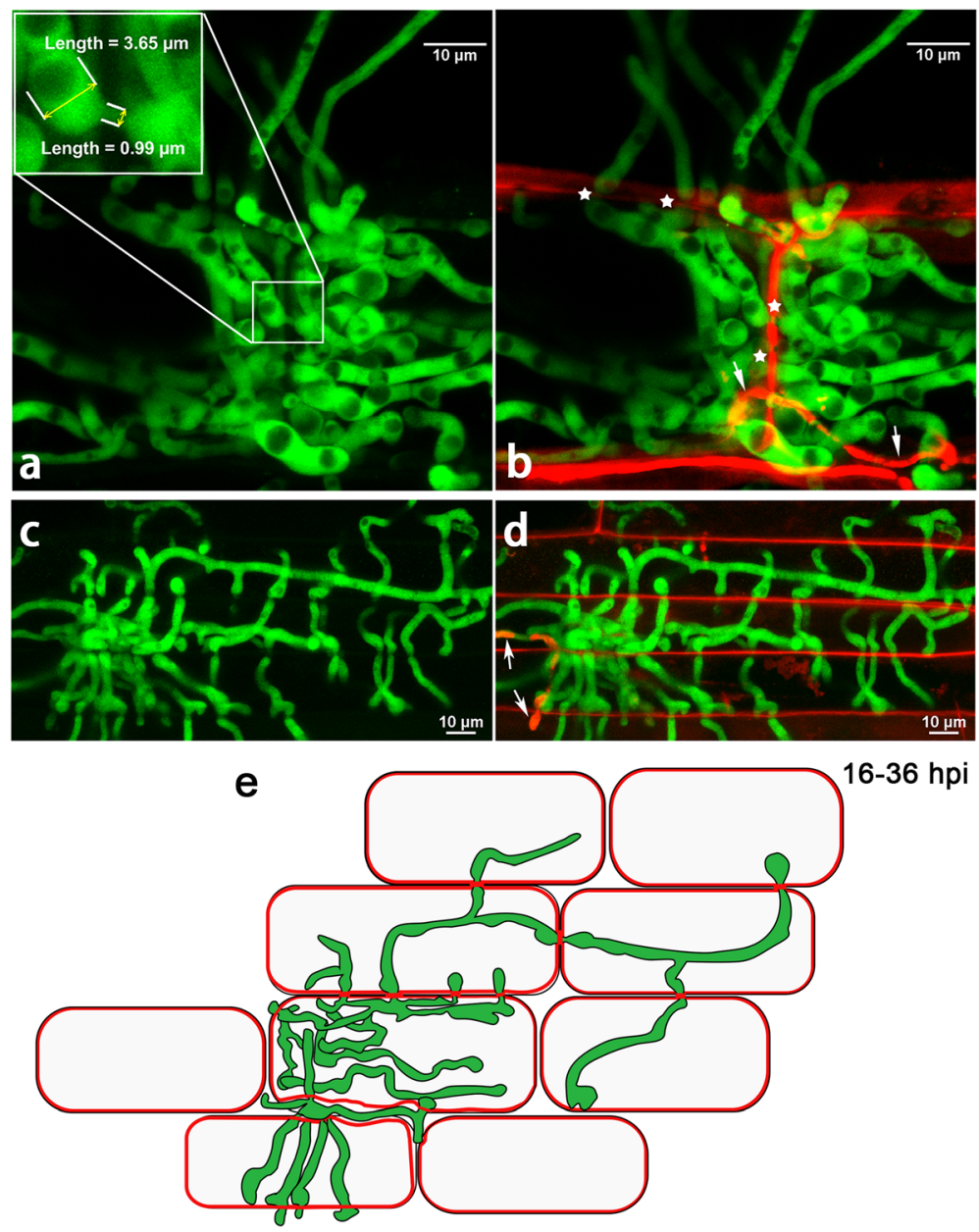

Fig. 4 Laser confocal microscopic images of F. graminearum infection of wheat coleoptiles at 24 hpi. Tissue cells of wheat coleoptiles were stained with PI (Propidium iodide) (red). a, b Cell-to-cell invasion using constricted invasive hyphae pegs (about $1 \mu \mathrm{m}$ ). This image is a threedimensional projection of 27 optical sections acquired with a z-interval of $0.35 \mu \mathrm{m}$. c, d Tissue cells of wheat coleoptiles were visible even after extensive proliferation of $F$. graminearum inside them. Contents of the infected cells are not disintegrated. PI staining identifies a few dead invasive hyphae (arrows) in the infected wheat coleoptiles cells at $24 \mathrm{hpi}$. This image is a three-dimensional projection of 55 optical sections acquired with a z-interval of $0.15 \mu \mathrm{m}$. e A proposed model depicting the early stages (16-36 hpi) of F. graminearum proliferation inside wheat coleoptiles

\section{Toxisome formation during fungal infection}

The mycotoxin-producing fungal pathogen F. graminearum is the causal agent of FHB in small grain cereals on the field worldwide (Desjardins et al. 1993; Goswami and Kistler 2004; Chen et al. 2019). Although F. graminearum pathogenesis has been widely investigated using molecular genetics approaches, studies on toxisome formation in wheat coleoptile cells at the initial stages of infection have not been conducted. To understand the spatial and temporal changes of toxisome upon induction in planta, we visualized the localization of Tri4-GFP which is known to be closely associated with toxisome structures in F. garminearum (Menke et al. 2013; Boenisch et al. 2017; Boenisch et al. 2019). Firstly, we investigated the dynamic changes of Tri4-GFP upon induction in an in vitro condition. Our results showed that the growing hyphae expressing the Tri4-GFP construct exhibit a reticulate fluorescence pattern on solid starch yeast media (SYM) (Fig. 7a), while they switch to crescent and spherical perinuclear pattern in liquid trichothecene biosynthesis (LTB) media (Fig. 7b), which is consistent with some previous findings (Boenisch et al. 2017; Tang et al. 2018). Next, we inoculated the strain expressing Tri4-GFP on wheat coleoptiles and performed time-course confocal microscopic observations. The results showed that the Tri4-GFP is poorly expressed in the invasive hyphae at early proliferation stage (12 hpi) (Fig. 7b). At 24 hpi, the Tri4-GFP strain demonstrated clear crescent spherical perinuclear structures in swollen invasive hyphae (Fig. 7b). At $36 \mathrm{hpi}$, the extensively grown fungal invasive hyphae were seen in the wheat coleoptile cells, some of which express the Tri4GFP signal (Fig. 7b). To analyze the expression pattern of 


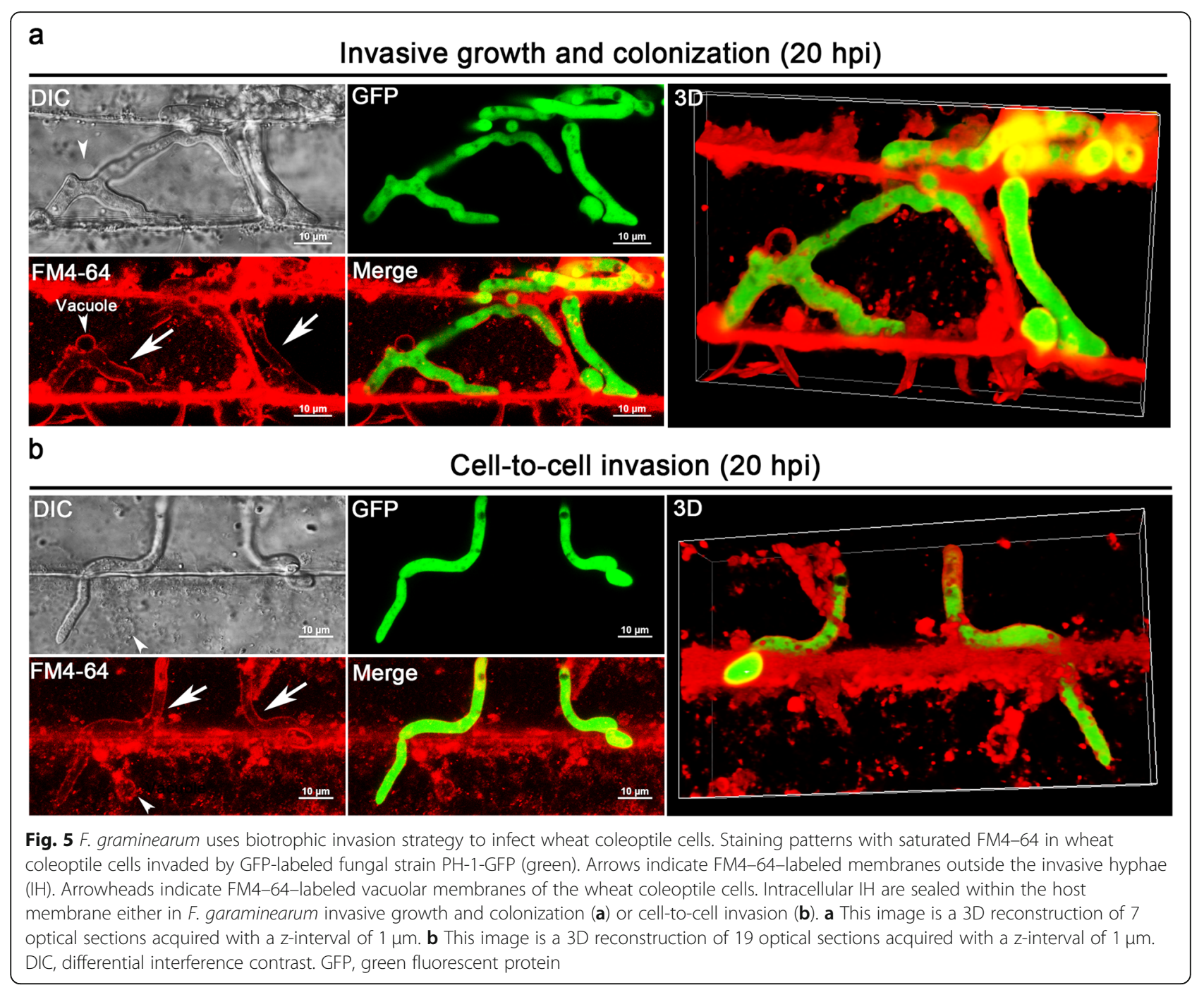

Tri4 in the wild-type, we extracted total RNA from wheat coleoptiles infected with Tri4-GFP strain at 12, 24, 36 and $48 \mathrm{~h}$ time-points. Real-time PCR revealed high relative expression levels of Tri4 at 24, 36 and $48 \mathrm{~h}$ at the mycotoxin induction stages as compared to $12 \mathrm{~h}$ (Fig. 7c). Thus, these results demonstrate that Tri4 activation requires external stimuli and is spatio-temporally modulated.

\section{Discussion}

This study revealed some new features of cellular mechanisms underlying plant disease caused by fungal pathogen. First, hyphae that differentiate into appressoria-like structures could play important role in initial penetration. Second, the infectious hyphae in the intercellular space become morphologically altered when they begin to invade the host cells. Third, high degree of hyphal plasticity in the intracellular invasive hyphae is responsible for successive cell penetration. Fourth, F. graminearum adopts biotrophic lifestyle for successful invasion of wheat coleoptile cells. Lastly, we developed a fluorescence imaging method to investigate host cellular reactions in relation to penetration and growth of $F$. graminearum in living tissues.

In plant pathogenic fungi, infection structures can be observed from the surface into the plant tissue and are important for breaching the outer surface of the host plant, thereby gaining entry into the internal tissues. Appressoria are specialized and typical infection structures that have been shown to be necessary for rusts, powdery mildews and blast diseases (Ryder and Talbot 2015; Talbot 2019). Previous studies have shown that $F$. graminearum does not usually require appressoria for host infection (Mendgen et al. 1996; Cuomo et al. 2007; Kikot et al. 2009). However, Boenisch and Schafer (2011) demonstrated that F. graminearum produces compound appressoria and foot structures on inoculated caryopses, paleas, lemmas and glumes of susceptible and resistant wheat cultivars during infection. 

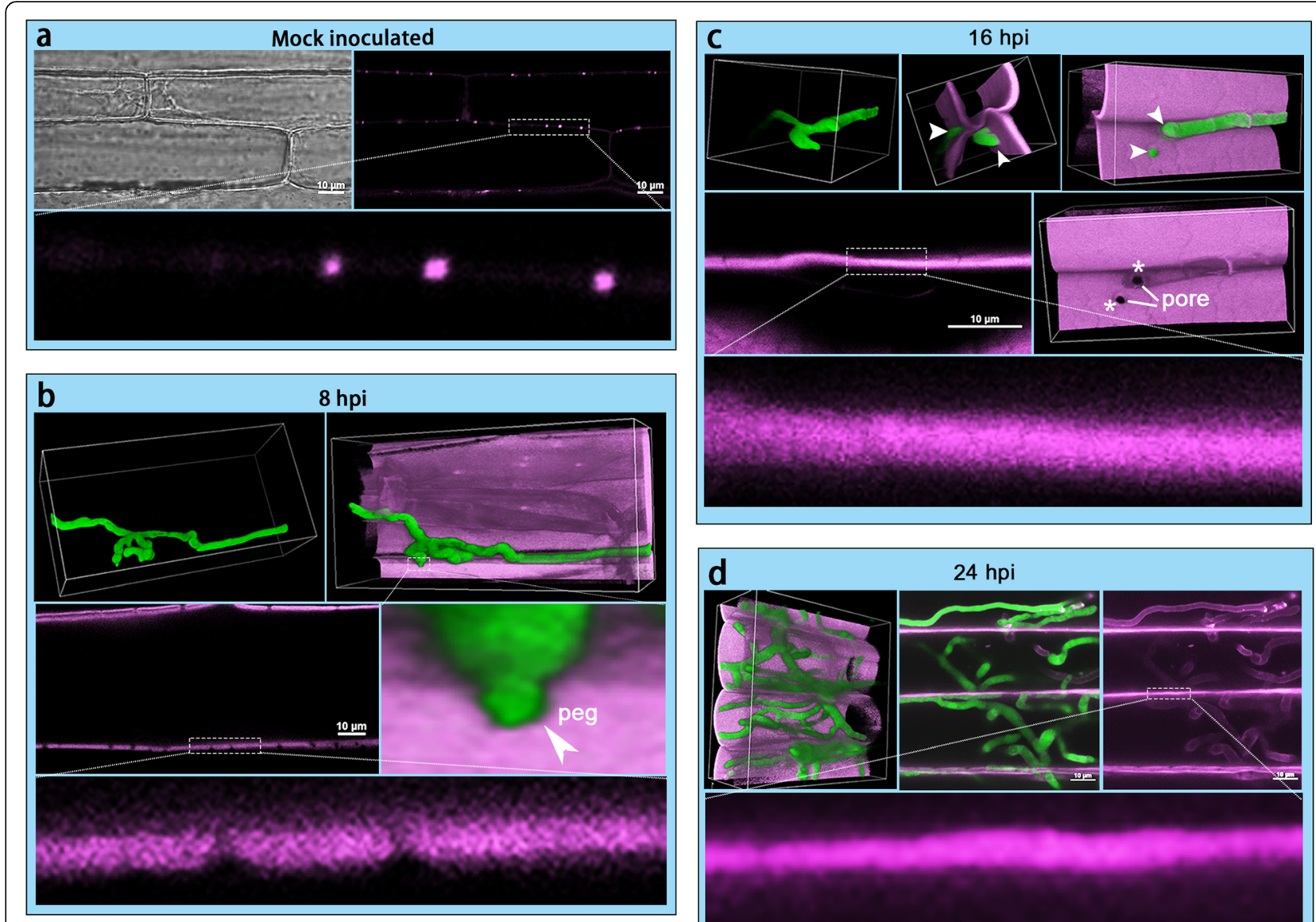

$24 \mathrm{hpi}$
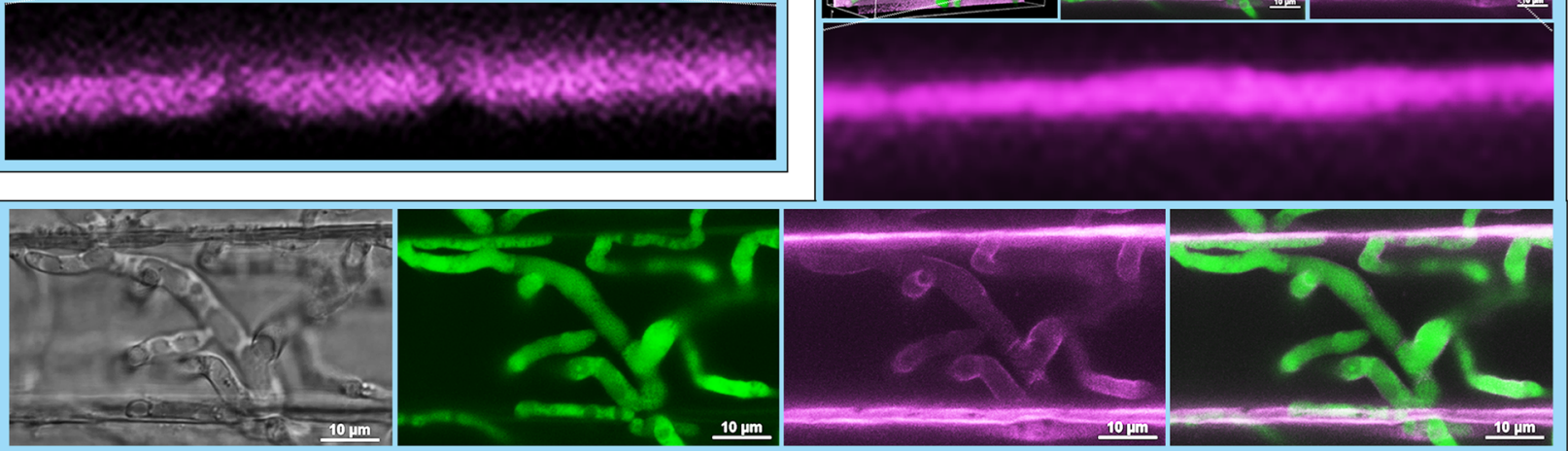

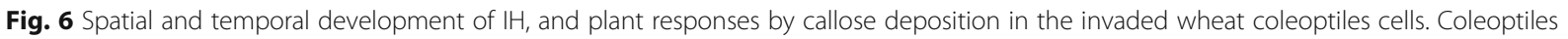
were stained with aniline blue and callose deposition was pictured at the indicated time-points. Detailed views of each boxed region are shown at the bottom of the respective images. a The mock-inoculated control coleoptile cells were poorly labeled with aniline blue, therefore showing limited discrete foci at the cell wall. b Callose signals formed continuous lines along the hyphal fronts before the swelling hyphae penetrate the wheat coleoptile cell wall (8 hpi) by means of a peg (arrowhead). This image is a 3D reconstruction of 101 optical sections acquired with a zinterval of $0.4 \mu \mathrm{m}$. c Callose accumulated dramatically when a hypha begins to traverse the host cell wall (16 hpi). Arrowheads indicate penetration sites. Asterisks indicate the pores due to peg penetration (without callose deposition). This image is a 3D reconstruction of 101 optical sections acquired with a z-interval of $0.3 \mu \mathrm{m}$. $\mathbf{d}$ Extensive fungal growth as seen in infected wheat coleoptiles cells at 24 hpi. Callose was not only deposited between the neighboring cell walls but also decorated at the outer linings of the invasive hyphae. This image is a 3D reconstruction of 128 optical sections acquired with a z-interval of $0.28 \mu \mathrm{m}$

The compound appressoria include lobate appressoria and infection cushions, which have highly branched and agglomerated hyphae, though the morphology of the foot structures is relatively simple (Boenisch and Schäfer 2011). In our study, we observed that after conidial germination and hyphal branching, multiple swollen hyphal structures of the fungus are formed and adhere tightly to the wheat coleoptile cell surfaces, which resemble the fungal foot structures (named here as appressoria-like structures) (Fig.
$2 \mathrm{a}, \mathrm{b})$. In addition, these appressoria-like structures were observed in the invasive hyphae during growth in the invaded wheat coleoptile cells (Fig. 4c, d). This is consistent with a previous observation that appressoria-like structures are formed in invaded wheat and barley epicarp cells (Jansen et al. 2005). In the rice-blast fungus $M$. oryzae, an appressorium generates enormous pressure of up to 8.0 $\mathrm{MPa}(80 \mathrm{~atm})$, which is used to physically rupture the host plant surface to gain entry into the host tissues (Dagdas et 


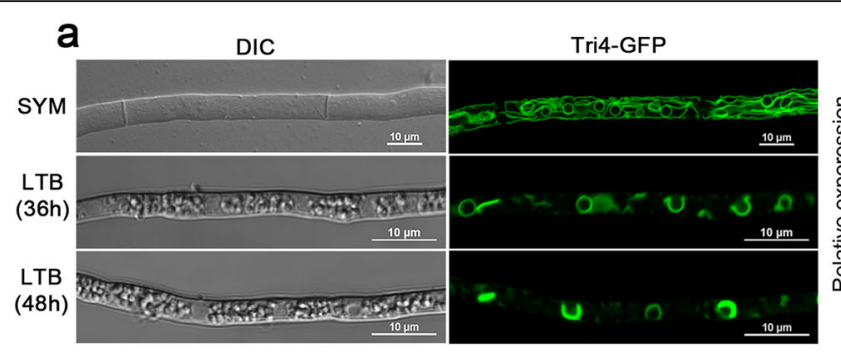

b

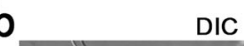

DIC

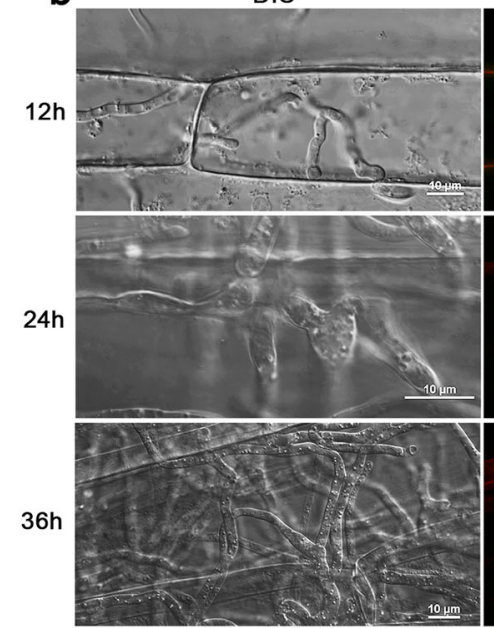

Tri4-GFP \& PI

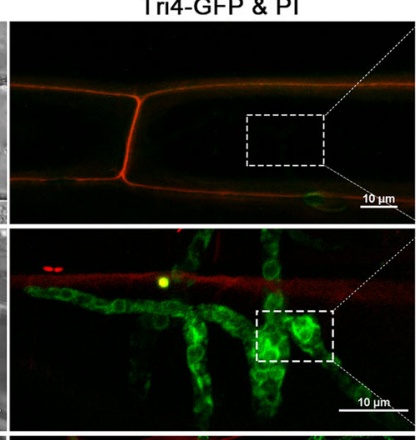

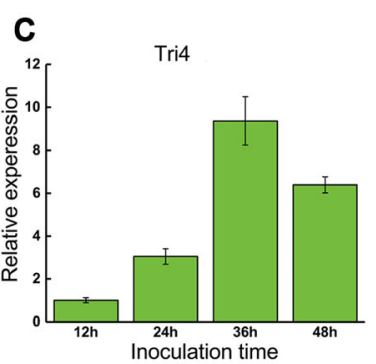

Detailed view

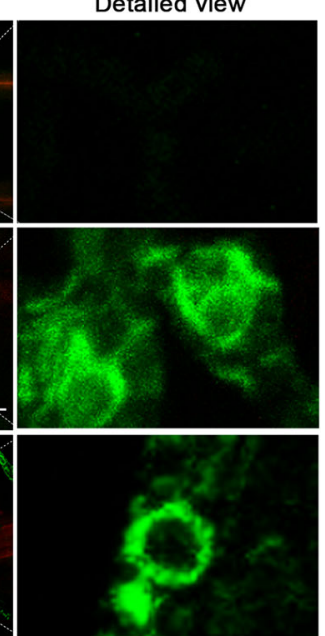

Fig. 7 Spatial and temporal changes of Tri4 upon induction in vitro and in planta. a The growing hyphae of Tri4-GFP strain exhibit a reticulate fluorescence pattern on solid starch yeast media (SYM), while it switches to crescent and spherical perinuclear pattern in liquid trichothecene biosynthesis (TBI) media. b The Tri4-GFP is poorly expressed in invasive hyphae at early proliferation stage (12 hpi). In swelling invasive hyphae, the Tri4-GFP develops clear crescent and spherical perinuclear structures at 24 hpi. At 36 hpi, extensively grown fungal invasive hyphae were seen in the wheat coleoptiles cells with some of these hyphae expressing Tri4-GFP. c Relative expression levels of Tri4 at the indicated time points after inoculation were determined by reverse transcriptase-quantitative polymerase chain reaction. Means \pm SE were calculated from the results of three replications

al. 2012; Talbot 2019). However, it is still not clear whether F. graminearum appressoria-like structures also utilize similar physical force to breach the outer barrier of its hosts. In $M$. oryzae, extensive cellular and molecular studies have been conducted to address the processes involved in appressorial formation and penetration (Wilson and Talbot 2009). A number of signaling pathways (including the cAMP, Pmk1 and Mps1 and MAP kinase pathways) are shown to be important for appressorial development and penetration (Wilson and Talbot 2009; Li et al. 2012); but it is still uncertain whether these signaling pathways play the same roles in appressoria-like structures of F. graminearum. Future studies to elucidate these questions will improve our understanding of the mechanisms of $F$. graminearum infection.

We also established that F. graminearum hyphae often colonize the intercellular spaces of the wheat coleoptile cells initially before proceeding into the cell (Fig. 2c), consistent with previous findings in epicarp cells of wheat and barley (Kang and Buchenauer 2000a; Brown et al. 2010). Importantly, we found that once the intercellular hyphae start to penetrate the host cell walls, they become bulbous and thick in appearance (Fig. 3c, d). In contrast, the morphology of the hyphae on the surface of wheat coleoptiles does not change prior to penetration (Fig. 3a, b). We reasoned that the hyphae colonizing the intercellular spaces of the host are relatively tougher than those growing on the epidermal cell surfaces due to confined nature of the intercellular spaces and strong immune responses from the host. The intercellular growth condition could also trigger, in F. graminearum, the secretion of some virulence factors or effectors to the host. Therefore, the surficial and intercellular hyphae use two different strategies, respectively, to penetrate the host barriers. Despite these two sets of hyphae having distinct morphological features, they use similar and highly constricted invasive hyphal pegs to cross the host cell walls and afterwards, the original hyphal diameter is restored (Fig. 3). In M. oryzae, these constricted invasive hyphal pegs were also observed in invaded cells and could be important for initial transition of the fungus through plasmodesmata (Kankanala et al. 2007). Plasmodesmata are known to facilitate the 
exchange of molecules between neighboring cells (Cheval and Faulkner 2018). To date, whether F. graminearum modulates its host plasmodesmata for successful penetration and/or cell-to-cell movement remains to be dissected.

Furthermore, there has not been a consensus about the biotrophic lifestyle of $F$. graminearum during the initial stages of host colonization (Jansen et al. 2005; Trail 2009; Brown et al. 2010; Kazan et al. 2012). Brown et al. (2010) found no indication of necrotrophy at those stages after infection as the advancing F. graminearum hyphae remained in the intercellular space of the wheat rachis cells before subsequent intracellular growth, which leads to cell death and necrosis. However, another group found that as soon as the pathogen enters the cytosol of the epicarp cells in both barley and wheat, plant cell death is induced, suggesting the absence of biotrophic lifestyle of the fungus at that stage (Jansen et al. 2005). In the present study, we first demonstrated by live cell imaging that the first- and later-invaded wheat coleoptile cells were still alive after colonization by F. graminearum (Fig. 4). To further confirm this, we stained these cells with FM4-64 and found that the biotrophic invasive hyphae of the fungus were separately sealed within a plant membrane as they colonized the living plant cells (Fig. 5). In addition, we demonstrate that the invasion pegs of the hyphae experience extreme constriction as they pierce the wheat cell walls (Figs. 3 and 4), which is a typical feature of biotrophic growth. Our results are consistent with an extended biotrophic invasion strategy reported in rice blast disease (Kankanala et al. 2007). This is also in line with a previous study which affirmed that F. graminearum initiates infection of coleoptiles using covert penetration strategies (Zhang et al. 2012).

As part of their defense responses, plants deposit callose polymer (composed of $1,3-\beta$-glucans) at penetration sites of invading pathogens (Blümke et al. 2014; Jia et al. 2019). In this study, we provide insights into the dynamics of callose deposition with respect to $F$. graminearum invasion. Interestingly, we observed the accumulation of callose not only on the wheat cell wall but also aligned on the outer linings of F. graminearum invasive hyphae (Fig. 6). This is an unexpected result and has not been reported before. We speculate that the callose deposition on the fungal invasive hyphae could act as the host strategy to slow pathogen invasion and effectively trigger the plant's innate immunity.

To suppress its host defense responses, F. graminearum produces mycotoxin and secretes it out to the host cells, causing tissue necrosis (Proctor et al. 1995; Trail 2009) so as to enable it spread into the rachis from the host florets (Jansen et al. 2005). However, our knowledge of spatiotemporal dynamics of toxisome formation by the pathogen at initial infection stages is limited. We therefore provide cellular and transcriptional evidences that the toxisome-related gene TRI4 is poorly expressed in the fungal invasive hyphae at early proliferation stage (12 hpi), which is consistent with the notion that these toxins are not necessary for the initial infection of wheat heads (Boenisch and Schäfer 2011. At later proliferation stages (24-36 hpi), typical crescent and spherical perinuclear structures were observed in the invasive hyphae, suggesting that deoxynivalenol (DON) mycotoxin was synthesized, leading to subsequent cell death and necrosis (Fig. 7b). Nevertheless, a previous study showed that almost no DON biosynthesis gene was induced during infection of coleoptiles (Zhang et al. 2012). We reasoned that transient injury by laser capture microdissection (used by the authors) might have affected the transcription and expression of toxisome-related genes. In addition, the different inoculation methods applied (in vitro and in vivo) could also result in this difference.

\section{Conclusions}

In summary, our analyses of the spatiotemporal nature of $F$. graminearum-wheat coleoptile interactions provide new insights into the penetration strategy of F. graminearum and the resulting host responses during the initial infection of the wheat. This paves the way for more detailed cytological investigations of $F$. graminearumwheat interactions which might help in establishing new way(s) of controlling FHB disease.

\section{Methods}

\section{Fungal strains and growth conditions}

We generated the F. graminearum transgenic strain $\mathrm{PH}-$ 1-GFP by transforming the plasmid pToxA-GFP into the wild-type strain PH-1 (Zheng et al. 2016). The PH-1GFP transformants were subsequently cultured on solid complete medium (CM) or starch yeast medium (SYM) at $25^{\circ} \mathrm{C}$. For conidia production, the $\mathrm{PH}-1-\mathrm{GFP}$ was grown in liquid carboxymethylcellulose culture (CMC) and conidia were harvested as described previously (Zheng et al. 2012). To visualize toxisome structures, the liquid trichothecene biosynthesis (LTB) were used as described previously (Menke et al. 2012).

\section{Infection assay}

Inoculation assays on wheat coleoptiles of the cultivar XiaoYan 22 were conducted as reported by Jia et al. (2017) with some minor modifications. 3-4 days old wheat seedlings were used. The wheat coleoptile was cut off from the bottom, and the enclosed leaves were removed. Next, outer green epidermis of the coleoptile was removed using a sterile surgical blade, but retaining the transparent inner epidermis for inoculation. These trimmed coleoptile tissue sections were 
mounted on microscope slides and $5 \mu \mathrm{L}$ conidia suspensions $\left(5 \times 10^{4}\right.$ spores $\left./ \mathrm{mL}\right)$ were dropped on them, respectively. The inoculated wheat coleoptiles were placed horizontally in Petri dishes containing wet filter papers to retain humid environments. When ready for microscopy, the surficial mycelia of $F$. graminearum on the wheat coleoptiles were gently scraped off with cotton swab and rinsed with water twice. Finally, the inoculated epidermis of the wheat coleoptile was stained and observed under a laser confocal microscope.

\section{Staining procedures}

Propidium iodide (PI, catalog No. P4170, $250 \mathrm{mg}$, Sigma) was dissolved in water to a stock concentration of $1 \mathrm{mg} /$ $\mathrm{mL}$. A working solution $(10 \mu \mathrm{g} / \mathrm{mL})$ of PI was prepared by dissolving $10 \mu \mathrm{L}$ of the stock solution in $1 \mathrm{~mL}$ of water. The infected wheat coleoptiles were immersed in the dye solution for $10 \mathrm{~min}$, and then observed under a microscope. $\mathrm{N}$-(3-Triethylammoniumpropyl)-4-(6-(4-(Diethylamino) Phenyl) Hexatrienyl) Pyridinium Dibromide (FM4-64, cata$\log$ No. T3166, $1 \mathrm{mg}$, ThermoFisher) was dissolved in water to a stock concentration of $0.5 \mathrm{mg} / \mathrm{mL}$. A working solution $(5 \mu \mathrm{g} / \mathrm{mL})$ of PI was prepared by dissolving10 $\mu \mathrm{L}$ of the stock solution in $1 \mathrm{~mL}$ of water. The infected wheat coleoptiles were immersed in the dye solution for $20 \mathrm{~min}$, and then observed under a microscope. The water-soluble aniline blue diammonium salt (catalog number: 415049, 25 g, Sigma-Aldrich) was dissolved to $0.1 \mathrm{mg} / \mathrm{mL}$ in $100 \mathrm{mM}$ $\mathrm{KH}_{2} \mathrm{PO}_{4}$ solution. The treated coleoptile was then immersed in the dye solution for $30 \mathrm{~min}$, and finally observed under a microscope.

\section{Confocal microscopy and image analysis}

Confocal microscopy was performed using a Nikon A1 inverted laser confocal microscope system (Tokyo, Japan). $405 \mathrm{~nm}$ light (Em. 452/45 nm) was used for aniline blue, GFP excitation was performed at $488 \mathrm{~nm}$ light (Em. 525/40 nm), PI and FM4-64 microscopies were performed at $561 \mathrm{~nm}$ light $(\mathrm{Em} .607 / 36 \mathrm{~nm})$. Images were processed using the Nikon A1 software (Tokyo, Japan). The resulting images were exported as avi files. Scanning was performed sequentially by firing the lasers for every channel to be used, in order to prevent crossexcitation.

\section{Additional files}

Additional file 1: Video S1 A hypha growing in the intercellular space of a wheat coleoptile cell. (AVI $2567 \mathrm{~kb}$ )

Additional file 2: Video S2 An extracellular hypha traversing the cell wall of a wheat coleoptile through a pit. (AVI 2617 kb)

Additional file 3: Video S3 Callose deposition at the cell wall of wheat coleoptiles after F. graminearum inoculation. (AVI 2822 kb)

\section{Abbreviations}

3D: Three dimension; CM: Complete medium; CMC: Carboxymethylcellulose; DON: Deoxynivalenol; F. graminearum: Fusarium graminearum; FHB: Fusarium head blight; FM4-64: N-(3-Triethylammoniumpropyl)-4-(6-(4-(Diethylamino) Phenyl) Hexatrienyl) Pyridinium Dibromide; GFP: Green fluorescent protein; hpi: Hours post inoculation; LTB: Liquid trichothecene biosynthesis; $M$. oryzae: Magnaporthe oryzae; PFT: Pore-forming toxin-like gene; PI: Propidium iodide; PM: Plasma membranes; SYM: Starch yeast media

\section{Acknowledgements}

We would like to thank Dr. Guanghui Wang (Northwest A\&F University), Huawei Zheng (Minjiang University) and Stefan Olsson (Fujian Agriculture and Forestry University) for fruitful discussions.

Authors' contributions

$H Q, X Z, W F$ and HW performed the experiments. All authors analyzed the data. WZ and ZW designed the study. WZ, XZ and YSA wrote the manuscript. All authors read and approved the final manuscript.

\section{Funding}

This study was funded by Natural Science Foundation of China (Grant No. 31772106, 31601596).

\section{Availability of data and materials}

The datasets used and/or analyzed during the current study are available from the corresponding author on reasonable request.

\section{Ethics approval and consent to participate}

Not applicable.

\section{Consent for publication}

Not applicable.

\section{Competing interests}

The authors declare that they have no competing interests.

\section{Author details}

${ }^{1}$ State Key Laboratory of Ecological Pest Control for Fujian and Taiwan Crops, College of Plant Protection, Fujian Agriculture and Forestry University, Fuzhou, China. ${ }^{2}$ Fujian Province Key Laboratory of Pathogenic Fungi and Mycotoxins, College of Life Sciences, Fujian Agriculture and Forestry University, Fuzhou, China. ${ }^{3}$ Department of Biochemistry, Faculty of Life Sciences, Ahmadu Bello University, Zaria, Nigeria. ${ }^{4}$ Institute of Oceanography, Minjiang University, Fuzhou, China.

Received: 3 July 2019 Accepted: 19 August 2019

Published online: 27 August 2019

\section{References}

Bai G, Shaner G. Management and resistance in wheat and barley to Fusarium head blight. Annu Rev Phytopathol. 2004;42:135-61

Blümke A, Falter C, Herrfurth C, Sode B, Bode R, Schäfer W, et al. Secreted fungal effector lipase releases free fatty acids to inhibit innate immunity-related callose formation during wheat head infection. Plant Physiol. 2014;165:346-58.

Boenisch MJ, Blum A, Broz KL, Gardiner DM, Kistler HC. Nanoscale enrichment of the cytosolic enzyme trichodiene synthase near reorganized endoplasmic reticulum in Fusarium graminearum. Fungal Genet Biol. 2019;124:73-7.

Boenisch MJ, Broz KL, Purvine SO, Chrisler WB, Nicora CD, Connolly LR, et al. Structural reorganization of the fungal endoplasmic reticulum upon induction of mycotoxin biosynthesis. Sci Rep. 2017;7:44296.

Boenisch MJ, Schäfer W. Fusarium graminearum forms mycotoxin producing infection structures on wheat. BMC Plant Biol. 2011;11:110.

Bolte S, Talbot C, Boutte Y, Catrice O, Read ND, Satiat-Jeunemaitre B. FM-dyes as experimental probes for dissecting vesicle trafficking in living plant cells. J Microsc. 2004;214:159-73

Brown NA, Urban M, van de Meene AM, Hammond-Kosack KE. The infection biology of Fusarium graminearum: defining the pathways of spikelet to spikelet colonisation in wheat ears. Fungal Biol. 2010;114:555-71.

Chen Y, Kistler HC, Ma Z. Fusarium graminearum trichothecene mycotoxins: biosynthesis, regulation, and management. Annu Rev Phytopathol. 2019;57. https://doi.org/10.1146/annurev-phyto-082718-100318. 
Cheval C, Faulkner C. Plasmodesmal regulation during plant-pathogen interactions. New Phytol. 2018;217:62-7.

Chowdhury J, Henderson M, Schweizer P, Burton RA, Fincher GB, Little A. Differential accumulation of callose, arabinoxylan and cellulose in nonpenetrated versus penetrated papillae on leaves of barley infected with Blumeria graminis f. sp. hordei. New Phytol. 2014;204:650-60.

Crowley LC, Scott AP, Marfell BJ, Boughaba JA, Chojnowski G, Waterhouse NJ. Measuring cell death by propidium iodide uptake and flow cytometry. Cold Spring Harb Protoc. 2016;7. https://doi.org/10.1101/pdb.prot087163.

Cuomo CA, Güldener U, Xu JR, Trail F, Turgeon BG, Di Pietro A, et al. The Fusarium graminearum genome reveals a link between localized polymorphism and pathogen specialization. Science. 2007;317:1400-2.

Dagdas YF, Yoshino K, Dagdas G, Ryder LS, Bielska E, Steinberg G, et al. Septin-mediated plant cell invasion by the rice blast fungus, Magnaporthe oryzae. Science. 2012;336:1590-5.

Dean R, Van Kan JA, Pretorius ZA, Hammond-Kosack KE, Di Pietro A, Spanu PD, et al. The top 10 fungal pathogens in molecular plant pathology. Mol Plant Pathol. 2012;13:414-30.

Desjardins AE, Hohn TM, McCormick SP. Trichothecene biosynthesis in Fusarium species: chemistry, genetics, and significance. Microbiol Rev. 1993;57:595-604.

Desjardins AE, Proctor RH. Molecular biology of Fusarium mycotoxins. Int J Food Microbiol. 2007;119:47-50.

Figueroa M, Hammond-Kosack KE, Solomon PS. A review of wheat diseases-a field perspective. Mol Plant Pathol. 2018;19:1523-36.

Goswami RS, Kistler HC. Heading for disaster: Fusarium graminearum on cereal crops. Mol Plant Pathol. 2004;5:515-25.

Jansen C, von Wettstein D, Schafer W, Kogel KH, Felk A, Maier FJ. Infection patterns in barley and wheat spikes inoculated with wild-type and trichodiene synthase gene disrupted Fusarium graminearum. Proc Natl Acad Sci U S A. 2005;102:16892-7.

Jia L-J, Tang H-Y, Wang W-Q, Yuan T-L, Wei W-Q, Pang B, et al. A linear nonribosomal octapeptide from Fusarium graminearum facilitates cell-to-cell invasion of wheat. Nat Commun. 2019;10:922.

Jia L-J, Wang W-Q, Tang W-H. Wheat coleoptile inoculation by Fusarium graminearum for large-scale phenotypic analysis. Bio-protocol. 2017;7:e2439.

Jones K, Kim DW, Park JS, Khang CH. Live-cell fluorescence imaging to investigate the dynamics of plant cell death during infection by the rice blast fungus Magnaporthe oryzae. BMC Plant Biol. 2016;16:69.

Kang Z, Buchenauer H. Cytology and ultrastructure of the infection of wheat spikes by Fusarium culmorum. Mycol Res. 2000a;104:1083-93.

Kang Z, Buchenauer H. Ultrastructural and immunocytochemical investigation of pathogen development and host responses in resistant and susceptible wheat spikes infected by Fusarium culmorum. Physiol Mol Plant Pathol. 2000b;57:255-68.

Kang Z, Zingen-Sell I, Buchenauer H. Infection of wheat spikes by Fusarium avenaceum and alterations of cell wall components in the infected tissue. Eur J Plant Pathol. 2005;111:19-28.

Kankanala P, Czymmek K, Valent B. Roles for rice membrane dynamics and plasmodesmata during biotrophic invasion by the blast fungus. Plant Cell. 2007:19:706-24

Kazan K, Gardiner DM, Manners JM. On the trail of a cereal killer: recent advances in Fusarium graminearum pathogenomics and host resistance. Mol Plant Pathol. 2012;13:399-413.

Kikot GE, Hours RA, Alconada TM. Contribution of cell wall degrading enzymes to pathogenesis of Fusarium graminearum: a review. J Basic Microbiol. 2009:49:231-41.

Li G, Zhou J, Jia H, Gao Z, Fan M, Luo Y, et al. Mutation of a histidine-rich calcium-binding-protein gene in wheat confers resistance to Fusarium head blight. Nat Genet. 2019;51:1106-12.

Li G, Zhou X, Xu JR. Genetic control of infection-related development in Magnaporthe oryzae. Curr Opin Microbiol. 2012;15:678-84.

Malinovsky FG, Fangel JU, Willats WG. The role of the cell wall in plant immunity. Front Plant Sci. 2014;5:178

McMullen M, Jones R, Gallenberg D. Scab of wheat and barley: a re-emerging disease of devastating impact. Plant Dis. 1997;81:1340-8.

Mendgen K, Hahn M, Deising H. Morphogenesis and mechanisms of penetration by plant pathogenic fungi. Annu Rev Phytopathol. 1996;34:367-86.

Menke J, Dong Y, Kistler HC. Fusarium graminearum Tri12p influences virulence to wheat and trichothecene accumulation. Mol Plant-Microbe Interact. 2012;25:1408-18.

Menke J, Weber J, Broz K, Kistler HC. Cellular development associated with induced mycotoxin synthesis in the filamentous fungus Fusarium graminearum. PLoS One. 2013;8:e63077.
Parry DW, Jenkinson P, McLeod L. Fusarium ear blight (scab) in small grain cereals - a review. Plant Pathol. 1995:44:207-38.

Peraldi A, Beccari G, Steed A, Nicholson P. Brachypodium distachyon: a new pathosystem to study Fusarium head blight and other Fusarium diseases of wheat. BMC Plant Biol. 2011;11:100.

Pritsch C, Muehlbauer GJ, Bushnell WR, Somers DA, Vance CP. Fungal development and induction of defense response genes during early infection of wheat spikes by Fusarium graminearum. Mol Plant-Microbe Interact. 2000;13:159-69.

Proctor RH, Hohn TM, McCormick SP. Reduced virulence of Gibberella zeae caused by disruption of a trichothecene toxin biosynthetic gene. Mol PlantMicrobe Interact. 1995;8:593-601.

Rawat N, Pumphrey MO, Liu S, Zhang X, Tiwari VK, Ando K, et al. Wheat Fhb1 encodes a chimeric lectin with agglutinin domains and a pore-forming toxin-like domain conferring resistance to Fusarium head blight. Nat Genet. 2016:48:1576-80

Rittenour WR, Harris SD. An in vitro method for the analysis of infection-related morphogenesis in Fusarium graminearum. Mol Plant Pathol. 2010;11:361-9.

Ryder LS, Talbot NJ. Regulation of appressorium development in pathogenic fungi. Curr Opin Plant Biol. 2015;26:8-13.

Skadsen RW, Hohn TM. Use of Fusarium graminearum transformed with gfp to follow infection patterns in barley and Arabidopsis. Physiol Mol Plant Pathol. 2004;64:45-53.

Stone BA, Evans NA, Bonig I, Clarke AE. The application of Sirofluor, a chemically defined fluorochrome from aniline blue for the histochemical detection of callose. Protoplasma. 1984;122:191-5.

Su Z, Bernardo A, Tian B, Chen H, Wang S, Ma H, et al. A deletion mutation in TaHRC confers Fhb1 resistance to Fusarium head blight in wheat. Nat Genet. 2019;51:1099-105.

Talbot NJ. Appressoria. Curr Biol. 2019;29:PR144-6.

Tang G, Chen Y, Xu J-R, Kistler HC, Ma Z. The fungal myosin I is essential for Fusarium toxisome formation. PLoS Pathog. 2018;14:e1006827.

Trail F. For blighted waves of grain: Fusarium graminearum in the postgenomics era. Plant Physiol. 2009;149:103-10.

Wilson RA, Talbot NJ. Under pressure: investigating the biology of plant infection by Magnaporthe oryzae. Nat Rev Microbiol. 2009;7:185-95.

Wu AB, Li HP, Zhao CS, Liao YC. Comparative pathogenicity of Fusarium graminearum isolates from China revealed by wheat coleoptile and floret inoculations. Mycopathologia. 2005;160:75-83.

Zhang XW, Jia L, Zhang Y, Jiang G, Li X, Zhang D, et al. In planta stage-specific fungal gene profiling elucidates the molecular strategies of Fusarium graminearum growing inside wheat coleoptiles. Plant Cell. 2012;24:5159-76.

Zhang Y, He J, Jia L, Yuan TL, Zhang D, Guo Y, et al. Cellular tracking and gene profiling of Fusarium graminearum during maize stalk rot disease development elucidates its strategies in confronting phosphorus limitation in the host apoplast. PLoS Pathog. 2016;12:e1005485.

Zheng W, Zhao X, Xie Q, Huang Q, Zhang C, Zhai H, et al. A conserved homeobox transcription factor Htf1 is required for phialide development and conidiogenesis in Fusarium species. PLoS One. 2012;7:e45432.

Zheng $W$, Zheng $H$, Zhao X, Zhang Y, Xie Q, Lin X, et al. Retrograde trafficking from the endosome to the trans-Golgi network mediated by the retromer is required for fungal development and pathogenicity in Fusarium graminearum. New Phytol. 2016;210:1327-43.

Ready to submit your research? Choose BMC and benefit from:

- fast, convenient online submission

- thorough peer review by experienced researchers in your field

- rapid publication on acceptance

- support for research data, including large and complex data types

- gold Open Access which fosters wider collaboration and increased citations

- maximum visibility for your research: over $100 \mathrm{M}$ website views per year

At BMC, research is always in progress.

Learn more biomedcentral.com/submissions 